\title{
Interacting Multiple Particle Filters For Fault Diagnosis of Non-linear Stochastic Systems
}

\author{
Xudong Wang and Vassilis L. Syrmos
}

\begin{abstract}
In this paper, an approach to fault diagnosis in a nonlinear stochastic dynamic system is proposed using the interacting multiple particle filtering (IMPF) algorithm. The fault diagnostic approach is formulated as a hybrid multiplemodel estimation scheme. The proposed diagnostic approach provides an integrated framework to estimate the system's current operational or faulty mode, as well as the unmeasured state variables in the system. Particle filtering algorithm is used to statistically model the underlying dynamics of a nonlinear/non-Gaussian stochastic system. A set of models is assumed to present the possible system behavior pattern or modes. A bank of particle filters runs in parallel, each based on a particular mode, to obtain mode-conditional estimates according to the probabilistically weighting scheme. The interaction among particle filters allows estimation from multiple filters to be fused in a principled manner. The simulation results on a highly nonlinear system are provided which demonstrate the effectiveness of the proposed method by comparing it with other nonlinear estimation techniques (extended Kalman filter (EKF) and unscented Kalman filter (UKF)-based).
\end{abstract}

\section{INTRODUCTION}

Due to the growing demand for fault tolerance, cost efficiency, high performance, improved reliability, availability, and enhanced safety, considerable research effort has been applied for the problem of fault detection and identification (FDI) in dynamic systems with ever increasing complexity. Different types of approaches have been both theoretically and experimentally investigated in $[1,2,3,4,5,6,7$, 8 , 14]. Such FDI techniques utilize parameter and state estimation, detection filters, statistical pattern recognition, multiple model estimator, maximum likelihood techniques and Bayes' theorem. These approaches fall into into two categories, i.e., model-based and data-driven approaches.

For model-based diagnostic approaches, appropriate mathematical models are assumed to be available to describe the system dynamics under different operational modes. The decision of a fault is based on available input and observation sequences and pre-determined system models. The system's operation is compared with predictions of a model and system operational mode is identified whenever predictions deviates from measurements. Physical parameters and estimation residuals are common features for modelbased diagnostics. Parameter estimation, observers (e.g., Kalman filtering and multiple model estimator) and parity space-based approaches are the main ways of generating

This research has been supported by the Office of Naval Research under contract N0014-05-02-0004.

$\mathrm{X}$. Wang is with the Research Corporation of University of Hawaii, Honolulu, HI 96822, USA xudongw@ hawaii .edu

V. L. Syrmos is with the Department of Electrical Engineering, University of Hawaii, Honolulu, HI 96822, USA syrmos@hawaii. edu the residuals. One of the most effective approaches for fault detection and identification is based on the multiple-model (MM) estimation scheme, in which a bank of filters runs in parallel, each based on a model matching to a particular operational mode. The diagnostic scheme uses the residual to compute mode-conditional likelihood function and determine the current active system operational mode based on the updated mode-conditional probabilities. Mode-conditional probabilities indicate the relative correctness of the various system mode. The overall state estimate is calculated by the probabilistically weighted sum of state estimates of all filters. MM algorithms for FDI have been developed for different engineering application problems [9, 10]. In [11], an interacting multiple model (IMM) algorithm was developed as a notable advance in the MM estimation. The IMM estimation introduces the interaction among single-model-based filters and thus leads to significantly improved performance for the hybrid system state estimation. The IMM algorithm has been applied for the detection and diagnosis of sensor and actuator failures [6] and the design of active fault-tolerant control [12]. Much of the development in model-based diagnostic approaches has relied on the assumption of linear system model and Gaussian noise and disturbances. By employing linearization techniques, diagnostic approaches developed for the linear case can be extended to some nonlinear stochastic systems. Extended Kalman filter is usually applied instead of Kalman filter for the nonlinear system diagnostic problem $[13,14]$. Another type of filter, called as unscented Kalman filter (UKF) [15] is also applicable for the nonlinear system estimation problem. Both EKF and UKF approximate the posterior distribution of the state as a Gaussian distribution. Although both EKF-based and UKF-based approaches seem perfectly straightforward, there are no general results to guarantee that such approximation will work in the most case. Often the FDI performance of these approaches suffer from poor detection or high false alarm rates.

With the motivation of IMM and particle filtering algorithms, a novel approach to detection and diagnosis of multiple faults in the nonlinear stochastic system is developed using the interacting multiple particle filtering algorithm. The diagnosis problem is to determine the state of a system over time given a stream of observations. The state being estimated is assumed to be hybrid, i.e., it consists of both discrete and continuous components. The system operational modes are described as the discrete components of the hybrid state, while the continuous components track the dynamic behavior of the system to be monitored. The modelbased diagnostic problem is to provide a distribution of the 
discrete state at each time step, while the dynamic state estimation problem is to construct the posterior probability density function of the continuous state on all the available information. By using the estimated distribution of system operational modes, a probabilistic scheme, such as maximum a posterior (MAP) algorithm can be designed to achieve both fault detection and diagnosis. Various estimates of the system's states can be obtained based on the estimated PDF of the continuous state. In the proposed fault diagnostic scheme, a system mode is drawn from a set of possible system modes. The evolution of the system mode over time is represented by a first order Markov chain. Particle filtering algorithm is applied to represent and recursively approximate the state conditional PDF. Each possible system mode is characterized with the corresponding particle filter. The transition and interaction among different system modes are described by the interacting multiple model algorithm. The proposed fault diagnostic approach can be applied for the FDI and dynamic state estimation problems in general nonlinear and non-Gaussian stochastic systems.

The rest of paper is organized as follows. Section II presents the FDI problem in general nonlinear and nonGaussian systems using multiple model method. In Section III the interacting multiple model algorithm is derived for general nonlinear and non-Gaussian systems. Section IV describes three different PDF approximation techniques using EKF, UKF and particle filtering approaches. In Section V, performance evaluation of the proposed diagnostic scheme is presented for a highly nonlinear system with conclusions in Section VI.

\section{PROBLEM STATEMENT}

With both the observation of system dynamic behavior and empirical knowledge of possible system modes available, the fault diagnosis problem is to determine the active system mode at each time step. In this section, multiple model-based estimation scheme is applied to formulate the fault diagnosis problem in the stochastic nonlinear system. In the MM method, a finite set of models is assumed to represent the possible system dynamic behavior; a bank of filters runs in parallel at every time to provide model-based estimates; the switching/transition between system modes is characterized by a first-order Markov chain.

In order to analyze and make inference about a dynamic system, at least two models are required: state transition and measurement model. The general state-space model can be broken down into those two models and presented in a probabilistic form as

$$
\begin{gathered}
p\left(x_{k} \mid x_{k-1}\right) \\
p\left(y_{k} \mid x_{k}\right),
\end{gathered}
$$

where $x_{k} \in \Re^{n_{x}}$ denotes the states (unobservable variables or parameters) of the system at time $k$ and $y_{k} \in \Re^{n_{y}}$ the observations. The system operational mode, $\theta_{k}$ is assumed to be drawn from a discrete set of modes, $\Theta=\left\{m_{1}, m_{2}, \cdots, m_{M}\right\}$. The transition probability between pairs of modes is denoted as

$$
P\left(\theta_{k+1}=m_{j} \mid \theta_{k}=m_{i}\right)=\pi_{i j} . \quad \forall m_{i}, m_{j} \in \Theta
$$

By defining the hybrid state as $\left\{x_{k}, \theta_{k}\right\}$, the probabilistic state-space model as equation (1) and (2) can be expressed as follows

$$
\begin{aligned}
x_{k} & =f_{\theta_{k}}\left(x_{k-1}, \theta_{k}, w_{k-1}\right) \\
y_{k} & =h_{\theta_{k}}\left(x_{k}, \theta_{k}, v_{k}\right),
\end{aligned}
$$

where $w_{k} \in \Re^{n_{w}}$ denotes the processing noise and $v_{k} \in \Re^{n_{v}}$ measurement noise. The mapping $f(\cdot)$ and $h(\cdot)$ represent the state evolution and state measurement model.

The fault diagnosis problem can be interpreted as the hybrid state estimation problem. State estimation is a process of determining the state of a system from a sequence of observations. Given that the probabilistic state-space formulation as equation (4) and (5), the Bayesian approach provides a rigorous framework for the hybrid state estimation problem. In the Bayesian approach, the posterior probability density function of the hybrid state as equation (6) is constructed based on the all available information (e.g.,observations, empirical knowledge of possible system modes).

$$
p\left(x_{k}, \theta_{k} \mid Y_{k}\right),
$$

where $Y_{k}$ is a sequence of system observation up to time $k, Y_{k}=\left\{y_{1}, \cdots, y_{k}\right\}$. Equation (6) incorporates the history of all available information and presents them statistically. If we know the posterior PDF, we can easily derive various estimates of the hybrid state. The fault diagnosis problem is to provide a distribution over the discrete set, $\Theta$ at each time step. This is the marginal distribution of the posterior PDF as

$$
p\left(\theta_{k} \mid Y_{k}\right)=\int p\left(x_{k}, \theta_{k} \mid Y_{k}\right) d x_{k} .
$$

Similarly, the problem of tracking the system dynamic behavior is to derive the marginal distribution of the posterior PDF as

$$
p\left(x_{k} \mid Y_{k}\right)=\sum_{i=1}^{M} p\left(x_{k} \mid \theta_{k}=m_{i}, Y_{k}\right) p\left(\theta_{k}=m_{i} \mid Y_{k}\right) .
$$

With the observation been updated at every time step, a recursive state estimation scheme can be designed. It is assumed that the initial PDF $p\left(x_{0}, \theta_{0}\right)$, also known as the prior, is available. The posterior PDF $p\left(x_{k}, \theta_{k} \mid Y_{k}\right)$ can be recursively estimated in two stages: prediction and update. Suppose that the desired posterior PDF $p\left(x_{k-1}, \theta_{k-1} \mid Y_{k-1}\right)$ at time $k-1$ is available. The prediction stage involves using the state transition model to obtain the predicted PDF $p\left(x_{k}, \theta_{k} \mid Y_{k-1}\right)$ of the hybrid state at time $\mathrm{k}$. By defining

$$
\rho_{k}=p\left(x_{k}, \theta_{k} \mid Y_{k}\right)
$$

the predicted PDF can be expressed as

$$
p\left(x_{k}, \theta_{k} \mid Y_{k-1}\right)=\int p\left(x_{k}, x_{k-1}, \theta_{k} \mid Y_{k-1}\right) d x_{k-1},
$$


where

$p\left(x_{k}, x_{k-1}, \theta_{k} \mid Y_{k-1}\right)=\sum_{\theta_{k-1}} p\left(x_{k} \mid x_{k-1}, \theta_{k}\right) p\left(\theta_{k} \mid \theta_{k-1}\right) \rho_{k-1}$.

The probabilistic model of the state transition $p\left(x_{k} \mid x_{k-1}, \theta_{k}\right)$ is defined by the state-space equation (4) and the known statistics of $w_{k}$.

At time step $k$, a new observation $y(k)$ becomes available and can be used to update the predicted PDF via Bayes' rule.

$$
p\left(x_{k}, \theta_{k} \mid Y_{k}\right)=\frac{p\left(y_{k} \mid x_{k}, \theta_{k}\right) p\left(x_{k}, \theta_{k} \mid Y_{k-1}\right)}{\int \sum_{\theta_{k}} p\left(y_{k} \mid x_{k}, \theta_{k}\right) p\left(x_{k}, \theta_{k} \mid Y_{k-1}\right) d x_{k}},
$$

where $p\left(y_{k} \mid x_{k}, \theta_{k}\right)$ is the mode-conditional likelihood function defined by the measurement model (5) and the known statistics of $v_{k}$.

Equation (9) and (10) form the basis for the recursive hybrid state estimation scheme. This recursive estimate scheme is only a conceptual solution and cannot be determined analytically. For the case with linear state transition and measurement models and Gaussian additive noise, analytical solutions to the recursive estimation of posterior PDF do exist. For such a special case, the posterior PDF can be summarized by the mean and covariance. The Kalman filter is often used to propagate the estimated mean and covariance.

For general nonlinear and non-Gaussian systems, the analytical solution is intractable. Nonlinear system linearization or PDF approximation techniques may provide alternative options to solve state estimation problems in the nonlinear system. Among those nonlinear system linearization techniques, the extended Kalman filter is the most popular approach to recursive nonlinear estimation [16]. The unscented Kalman filter is a method to approximate the non-Gaussian distribution by capturing the posterior mean and covariance accurately to the $2^{\text {nd }}$ order, with error only introduced in the $3^{\text {rd }}$ and higher orders [15]. With the great advantage of the particle filtering algorithm, the particle filtering techniques approximate posterior PDF using a set of weighted particles. This allows for a complete representation of the posterior PDF of the states. They can therefore, handle any nonlinearities or distribution [17].

\section{NONLINEAR INTERACTING MULTIPLE MODEL ALGORITHM}

Before we proceed to the detailed distribution approximation, the interacting multiple model algorithm is introduced to the recursive state estimation scheme. The IMM method is the extension of the conventional MM approach by introducing the interacting scheme among different modes and the first-order Markov chain to describe the switching/transition between modes. Unlike the MM method, in which the singlemodel-based filters are running in parallel without mutual interaction, the interacting scheme of the IMM method reinitializes each filter by mixing the all most recent estimates from the single-model-based filters. The proposed recursive state estimation scheme can be broke down into three stages: re-initialization, prediction and update.
TABLE I

ONE CYCLE OF RECURSIVE STATE ESTIMATION FOR $j^{\text {TH }}$ MODE-BASED FILTER

1. Prediction stage
$p\left(x_{k} \mid \theta_{k}=m_{j}, Y_{k-1}\right)=\int p\left(x_{k} \mid x_{k-1}, \theta_{k}=m_{j}\right) \rho_{k-1}^{j, 0} d x_{k-1}$,
where $\rho_{k-1}^{j, 0}=p\left(x_{k-1} \mid \theta_{k}=m_{j}, Y_{k-1}\right)$.
2. Update stage
$p\left(x_{k} \mid \theta_{k}=m_{j}, Y_{k}\right)=\frac{L_{k}^{j} p\left(x_{k} \mid \theta_{k}=m_{j}, Y_{k-1}\right)}{\int L_{k}^{j} p\left(x_{k} \mid \theta_{k}=m_{j}, Y_{k-1}\right) d x_{k}}$,
where $L_{k}^{j}=p\left(y_{k} \mid x_{k}, \theta_{k}=m_{j}\right)$.
3. Mode-conditional probability
$\mu_{k}^{j}=\frac{\mu_{k \mid k-1}^{j} \int L_{k}^{j} p\left(x_{k} \mid \theta_{k}=m_{j}, Y_{k-1}\right) d x_{k}}{\sum_{i} \mu_{k \mid k-1}^{i} \int L_{k}^{i} p\left(x_{k} \mid \theta_{k}=m_{i}, Y_{k-1}\right) d x_{k}}$.

By defining

$$
\mu_{k}^{j}=p\left(\theta_{k}=m_{j} \mid Y_{k}\right)
$$

and

$$
\mu_{k \mid k-1}^{j}=p\left(\theta_{k}=m_{j} \mid Y_{k-1}\right)=\sum_{i=1}^{M} \pi_{i j} \mu_{k-1}^{i},
$$

the mode-based re-initialization can be described as a mixing process as

$$
p\left(x_{k-1} \mid \theta_{k-1}, Y_{k-1}\right) \quad \overrightarrow{\text { Mixing }} \quad p\left(x_{k-1} \mid \theta_{k}, Y_{k-1}\right) .
$$

For the $j^{\text {th }}$ mode-based filter, the initial distribution estimation can be expressed as

$$
p\left(x_{k-1} \mid \theta_{k}=m_{j}, Y_{k-1}\right)=\frac{\sum_{i=1}^{M} \mu_{k-1}^{i} \pi_{i j} \rho_{k-1}^{i}}{\sum_{l=1}^{M} \pi_{l j} \mu_{k-1}^{l}},
$$

where $\rho_{k-1}^{i}=p\left(x_{k-1} \mid \theta_{k-1}=m_{i}, Y_{k-1}\right)$.

One cycle of recursive state estimation is illustrated in Table I. Using the law of total probability, the unconditional posterior state distribution can estimated using weighted mode-conditional posterior distribution estimation as

$$
p\left(x_{k} \mid Y_{k}\right)=\sum_{i} p\left(x_{k} \mid \theta_{k}=m_{i}, Y_{k}\right) \mu_{k}^{i} .
$$

As we can see from the total posterior distribution (15), the overall state estimate is the probabilistically weighted modelbased estimates. The mode-conditional probabilities provide an indication of mode in effect at any time. It is natural to be used as an indication of a fault occurrence. By using the information from the mode-conditional probabilities, both fault detection and identification can be achieved. The maximum a posterior (MAP) is used to design the FDI scheme.

$\mu_{k}^{j}=\max _{i} \mu_{k}^{i}= \begin{cases}>\mu_{T} & \rightarrow m_{j}: \text { mode } \mathrm{j} \text { occurred } \\ <\mu_{T} & \rightarrow m_{1}: \text { nominal mode occurred }\end{cases}$

where $\mu_{T}$ is the fault decision threshold. Figure 1 shows the nonlinear interacting multiple model-based estimation scheme. 


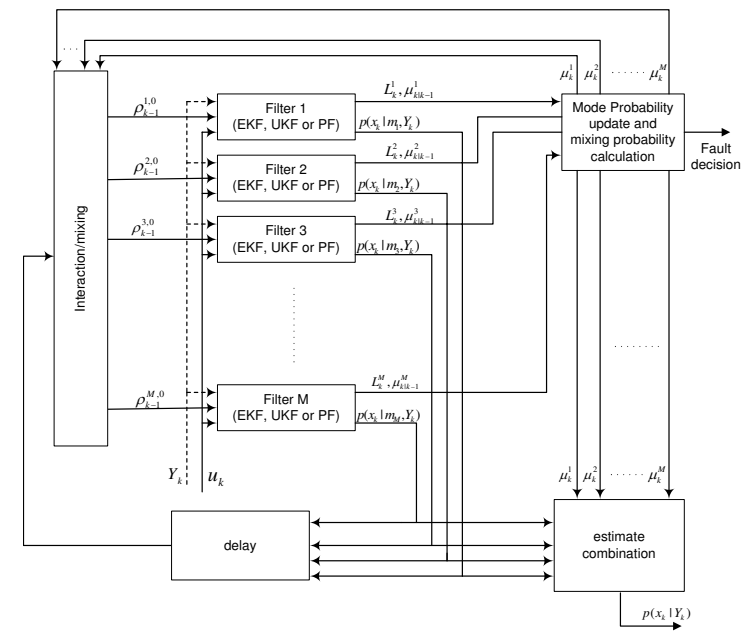

Fig. 1. Block diagram of the nonlinear IMM-based estimation scheme

\section{FDI VIA EKF, UKF AND PARTICLE FILTERING}

In this section, we present the EKF, UKF and particle filtering to approximate the posterior state distribution. For both EKF and UKF methods, the posterior distribution is approximated to be a Gaussian distribution, only the mean and covariance of the state distribution are recursively propagated and updated. The particle filtering algorithm uses a set of weighted particles to approximate the posterior.

The EKF is a minimum mean-square-error (MMSE) estimator based on the Taylor series expansion of the nonlinear functions $f(\cdot)$ and $h(\cdot)$ around the state estimate $\hat{x}_{k \mid k-1}$ of $x_{k}$. The following recursive estimation scheme for the mean $\hat{x}$ and covariance $P$ of the Gaussian approximation.

$$
\begin{aligned}
\hat{x}_{k \mid k-1} & =f\left(\hat{x}_{k-1}\right) \\
P_{k \mid k-1} & =F_{k} P_{k-1} F_{k}^{T}+G_{k} Q_{k} G_{k}^{T} \\
K_{k} & =P_{k \mid k-1} H_{k}^{T}\left[U_{k} R_{k} U_{k}^{T}+H_{k}+P_{k \mid k-1} H_{k}^{T}\right]^{-1} \\
\hat{x}_{k} & =\hat{x}_{k \mid k-1}+K_{k}\left(y_{k}-h\left(\hat{x}_{k \mid k-1}\right)\right) \\
P_{k} & =P_{k} \mid k-1-K_{k} H_{k} P_{k \mid k-1},
\end{aligned}
$$

where $K_{k}$ is the Kalman gain, $Q$ is the variance of the processing noise with zero mean, and $R$ is the variance of the measurement noise with zero mean. $F_{k}=\left.\frac{\partial f}{\partial x_{k}}\right|_{x_{k}=\hat{x}_{k \mid k-1}}$ and $G_{k}=\left.\frac{\partial f}{\partial w_{k}}\right|_{w_{k}=\hat{w}}$ are the Jacobian matrices of the state evolution model. $H_{k}=\left.\frac{\partial h}{\partial x_{k}}\right|_{x_{k}=\hat{x}_{k \mid k-1}}$ and $U_{k}=\left.\frac{\partial h}{\partial v_{k}}\right|_{v_{k}=\hat{v}}$ are the Jacobian matrices of the state measurement model. The interacting multiple EKF-based estimation scheme can be implemented by simply designing the bank of filters using EKF.

The unscented Kalman filter is a recursive MMSE estimator, in which the state distribution is also approximated as a Gaussian distribution, a minimal set of deterministic sample points are chosen to capture the true mean and covariance and are propagated through the nonlinear system. The complete UKF algorithm to approximate the posterior distribution of the state is given as Table II. $L$ is the dimension of the augmented state $x^{a}$, and $W_{i}^{c, m}$ are the weights [15]. Similarly, the interacting multiple UKF-based
TABLE II

ONE CYCLE OF RECURSIVE ESTIMATION USING UKF

1. Initialization with prior as $x_{0} \sim N\left(\hat{x}_{0}, P_{0}\right)$
$\hat{x}_{0}^{a}=\left(\begin{array}{lll}\hat{x}_{0}^{T} \quad \mathbf{0} & \mathbf{0}\end{array}\right)^{T}, \quad P_{0}^{a}=\left(\begin{array}{ccc}P_{0} & \mathbf{0} & \mathbf{0} \\ \mathbf{0} & Q & \mathbf{0} \\ \mathbf{0} & \mathbf{0} & R\end{array}\right)$
2. Calculate the sigma points
$\chi_{k-1}^{a}=\left(\begin{array}{l}\hat{x}_{k-1}^{a} \quad \hat{x}_{k-1}^{a} \pm \sqrt{\left(n_{a}+\lambda\right) P_{k-1}^{a}}\end{array}\right)$
3. State prediction based on the propagation of sigma points
$\quad \chi_{k \mid k-1}^{a}=f\left(\chi_{k-1}^{a}\right), \quad \hat{x}_{k \mid k-1}=\sum_{i=0}^{2 L} W_{i}^{m} \chi_{i, k \mid k-1}^{x, a}$
$P_{k \mid k-1}=\sum_{i=0}^{2 L} W_{i}^{c}\left[\chi_{i, k \mid k-1}^{x, a}-\hat{x}_{k \mid k-1}\right]\left[\chi_{i, k \mid k-1}^{x, a}-\hat{x}_{k \mid k-1}\right]^{T}$
$\Upsilon_{k \mid k-1}=h\left(\chi_{k \mid k-1}^{a}\right), \quad \hat{y}_{k \mid k-1}=\sum_{i=0}^{2 L} W_{i}^{m} \Upsilon_{i, k \mid k-1}$
3. Measurement updates $\quad$
$P_{y_{k}}=\sum_{i=0}^{2 L} W_{i}^{c}\left[\Upsilon_{i, k \mid k-1}-\hat{y}_{k \mid k-1}\right]\left[\Upsilon_{i, k \mid k-1}-\hat{y}_{k \mid k-1}\right]^{T}$
$P_{x_{k} y_{k}}=\sum_{i=0}^{2 L} W_{i}^{c}\left[\chi_{i, k \mid k-1}^{x, a}-\hat{x}_{k \mid k-1}\right]\left[\Upsilon_{i, k \mid k-1}-\hat{y}_{k \mid k-1}\right]^{T}$
$K_{k}=P_{x_{k} y_{k}} P_{y_{k}}^{-1}, \quad P_{k}=P_{k \mid k-1}-K_{k} P_{y_{k}} K_{k}^{T}$
$\hat{x}_{k}=\hat{x}_{k \mid k-1}+K_{k}\left(y_{k}-\hat{y}_{k \mid k-1}\right)$

estimation scheme can be implemented by designing the filters using UKF.

The basic idea of particle filtering is to use a random measure $\left\{x_{k}^{i}, w_{k}^{i}\right\}_{i=1}^{N}$ to characterize the posterior distribution of the state, $p\left(x_{k} \mid Y_{k}\right)$, where $\left\{x_{k}^{i}\right\}_{i=1}^{N}$ is a set of support points with associated weights $\left\{w_{k}^{i}\right\}_{i=1}^{N}$. The weights are normalized such that $\sum_{i} w_{k}^{i}=1$. Then the posterior density at time $k$ can be approximated as

$$
p\left(x_{k} \mid Y_{k}\right) \approx \sum_{i=1}^{N} w_{k}^{i} \delta\left(x_{k}-x_{k}^{i}\right) .
$$

By applying the particle filter-based density approximation, a complete cycle of distribution estimation can be described as Table III. As we can see, the predicted state distribution can be approximated with a set of weighted kernel density distribution, $\left\{\hat{w}_{k-1}^{l, i}, p\left(x_{k} \mid x_{k-1}^{l, i}, \theta_{k}=m_{j}\right)\right\}$. The weight $w_{k-1}^{l, i}$ is the indication of the contribution of each kernel density to the predicted density. $N$ particles, $\left\{x_{k \mid k-1}^{i, j}\right\}_{i=1}^{N}$ are sampled from the set of kernel density functions, with particles sampled from each kernel being determined according to the corresponding kernel weights. After sampling, the predicted state distribution can be approximated with a new set of weighted particles, $\left\{w_{k \mid k-1}^{i, j}, x_{k \mid k-1}^{i, j}\right\}_{i=1}^{N}$.

\section{NUMERICAL EXAMPLE AND SIMULATION RESULTS}

In this section, the proposed interacting multiple particle filtering algorithms is applied for the hybrid state estimation problem in a highly nonlinear system. The performance on the fault diagnosis and state estimation is evaluated through Monte Carlo simulation experiments and the results are compared with that using EKF and UKF-based approach.

The highly nonlinear system is a univariate growth model [18] as

$$
\begin{aligned}
& x_{k}=a x_{k-1}+b \frac{x_{k-1}}{1+x_{k-1}^{2}}+8 \cos (1.2(k-1))+w_{k-1} \\
& y_{k}=0.5 x_{k}^{2}+v_{k},
\end{aligned}
$$


TABLE III

ONE CYCLE OF RECURSIVE ESTIMATION FOR $j^{\text {th }}$ PARTICLE FILTER

$$
\begin{aligned}
& \text { 1. Initialization } \\
& p\left(x_{k-1} \mid \theta_{k}=m_{j}, Y_{k}\right) \approx \sum_{i=1}^{M} \sum_{l=1}^{N} \hat{w}_{k-1}^{l, i} \delta\left(x_{k-1}-x_{k-1}^{l, i}\right), \\
& \hat{w}_{k-1}^{l, i}=\frac{w_{k-1}^{l, i} \mu_{k-1}^{i} \pi_{i j}}{\sum_{m} \pi_{m j} \mu_{k-1}^{j}}, \\
& \left\{w_{k-1}^{l, i}, x_{k-1}^{l, i}\right\} \text { is the set of particles from } i^{\text {th }} \text { particle filter. } \\
& \text { 2. Prediction using evolution model } \\
& p\left(x_{k} \mid \theta_{k}=m_{j}, Y_{k-1}\right) \approx \sum_{i=1}^{M} \sum_{l=1}^{N} \hat{w}_{k-1}^{l, i} \rho_{k \mid k-1, j}^{l, i}, \\
& \rho_{j, k \mid k-1}^{l, i}=p\left(x_{k} \mid x_{k-1}^{l, i}, \theta_{k}=m_{j}\right) . \\
& \text { Sample } N \text { weighted particles as }\left\{w_{k \mid k-1}^{i, j}, x_{k \mid k-1}^{i, j}\right\}_{i=1}^{N} . \\
& p\left(x_{k} \mid \theta_{k}=m_{j}, Y_{k-1}\right) \approx \sum_{i=1}^{N} w_{k \mid k-1}^{i, j} \delta\left(x_{k}-x_{k \mid k-1}^{i, j}\right), \\
& w_{k}^{i, j}=\frac{w_{k \mid k-1}^{i, j}}{\sum_{l=1}^{N} w_{k \mid k-1}^{l, j} p\left(y_{k} \mid x_{k \mid k-1}^{l, j}, \theta_{k}=m_{j}\right)} . \\
& \text { 3. Update } \\
& p\left(x_{k} \mid \theta_{k}=m_{j}, Y_{k}\right) \approx \sum_{i=1}^{N} w_{k}^{i, j} \delta\left(x_{k}-x_{k \mid k-1}^{i, j}\right), \\
& w_{k}^{i, j}=\frac{w_{k \mid k-1}^{i, j}}{\sum_{l=1}^{N} w_{k \mid k-1}^{l, j} p\left(y_{k} \mid x_{k \mid k-1}^{l, j}, \theta_{k}=m_{j}\right)} . \\
& \text { 4. Resample as [17], assign weight to each particle as } w_{k}^{i, j}=\frac{1}{N} . \\
& \text { 5. Mode-conditional probability update } \\
& \mu_{k}^{j} \approx \frac{\mu_{k \mid k-1}^{j} \sum_{l=1}^{N} w_{k \mid k-1}^{l, j} p\left(y_{k} \mid x_{k \mid k-1}^{l, j}, \theta_{k}=m_{j}\right)}{\sum_{i=1}^{M} \sum_{l=1}^{N} \mu_{k \mid k-1}^{i} w_{k \mid k-1}^{l, i} p\left(y_{k} \mid x_{k \mid k-1}^{l, i}, \theta_{k}=m_{i}\right)} \\
& \text { 6. Compute overall state estimate } \\
& \hat{x}_{k}=\sum_{j=1}^{M} \sum_{i=1}^{N} \mu_{k}^{j} w_{k}^{i, j} x_{k}^{i, j} \text {. } \\
&
\end{aligned}
$$

where $w_{k}$ and $v_{k}$ are uncorrelated zero mean Gaussian white noise with variance $Q_{w}=0.1$ and $Q_{v}=1$. Parameters $a$ and $b$ are $a=a_{0}=0.5$ and $b=b_{0}=25$. For the particle filtering-based approach, the number of particle is set to $N=200$. EKF, UKF and particle filter are initiated with the prior distribution, $p\left(x_{0}\right) \sim N(0,2)$. The fault is simulated as that the parameter $b$ jumps from a value of $b_{0}$ to $0.5 b_{0}$ while parameter $a$ remains unchanged. The fault is introduced at time $k=301$. The switching/transition probabilities between nominal mode and faulty mode are described by the transition probability matrix $H_{T}$.

$$
H_{T}=\left(\begin{array}{cc}
0.9 & 0.1 \\
0.01 & 0.99
\end{array}\right)
$$

EKF and UKF-based, and particle filtering algorithms are applied for the interacting multiple model-based hybrid state estimation. To assess the FDI performance of the proposed algorithm, Monte Carlo simulation experiments have been carried out. The performance indices considered here are the false alarm (FA) and the missed detection (MD) rate given the decision threshold $\mu_{T}$. The detailed definition of performance indices is referred to [12].

A set of fault decision threshold is designed as $\mu_{T} \in$ $\{0.7,0.95\}$ with the incremental step as 0.01 . Fifty Monte Carlo simulations are generated for each chosen fault decision threshold in each filtering algorithm. The FA and MD for each fault decision threshold in each filtering algorithm are shown in Figure 2 and 3 respectively. It is obviously desirable to design the recursive estimation scheme with low FA and MD rates. The FA and MD rates in the particle filtering-based approach are much lower than those

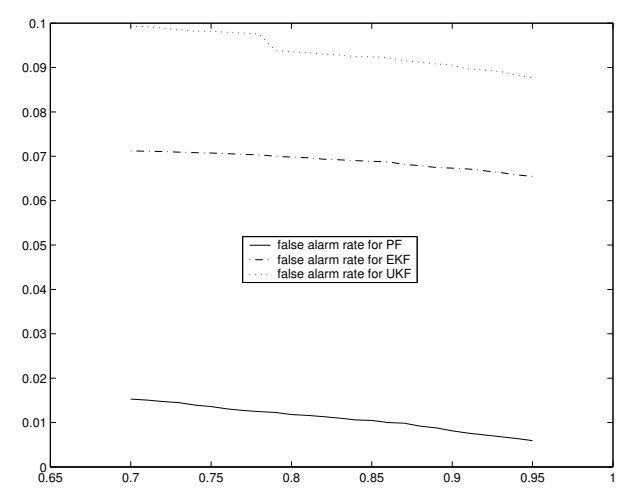

Fig. 2. False alarm rate for EKF, UKF and particle filtering-based approaches

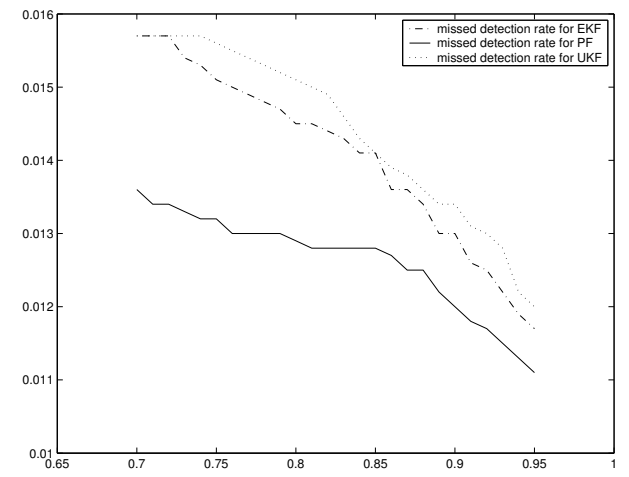

Fig. 3. Miss detection rate for EKF, UKF and particle filtering-based approaches

of the EKF and UKF-based approaches. The overall state estimation and estimation error are shown in Figure 4. The mode-conditional probabilities at each time step in each filtering algorithm are demonstrated in Figure 5, in which $P_{n}$ denotes the normal mode-conditional probability while $P_{s}$ fault mode-conditional probability. As we can see, the particle filtering-based approach provides more clear fault decision than EKF-based and UKF-based approaches.

Performance of the proposed particle filtering-based approach with different sample size is compared in Figure 6. One sample size is 200 while the other is 500. As we can see from the comparison of state estimation and modeconditional probabilities, the proposed particle filtering-based approach with less sample size can achieve almost same performance as the one with bigger sample size, in which more computation load are required.

\section{CONCLUSIONS}

An interacting multiple particle filtering algorithm has been applied for the fault diagnosis and state estimation problem in the nonlinear stochastic dynamic system. Its performance of fault diagnosis and estimation was evaluated through Monte Carlo simulations and compared with that using extend Kalman filtering or unscented Kalman filtering. The proposed fault diagnosis approach can not only recursively estimate the mode-conditional probability so that the active system mode at each time step can be 

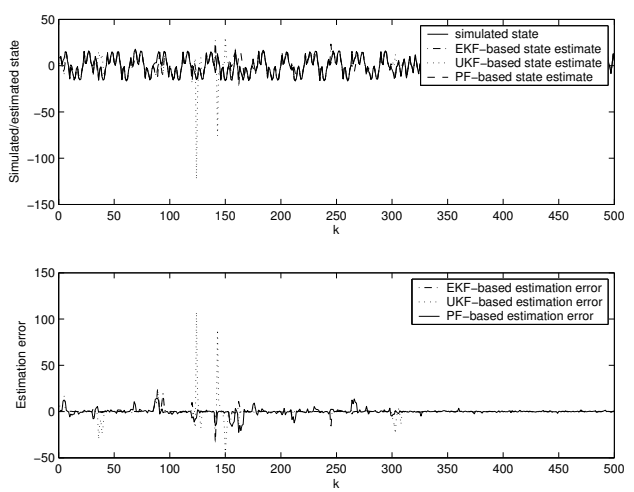

Fig. 4. Overall state estimate and estimation error for EKF, UKF and particle filtering-based approaches
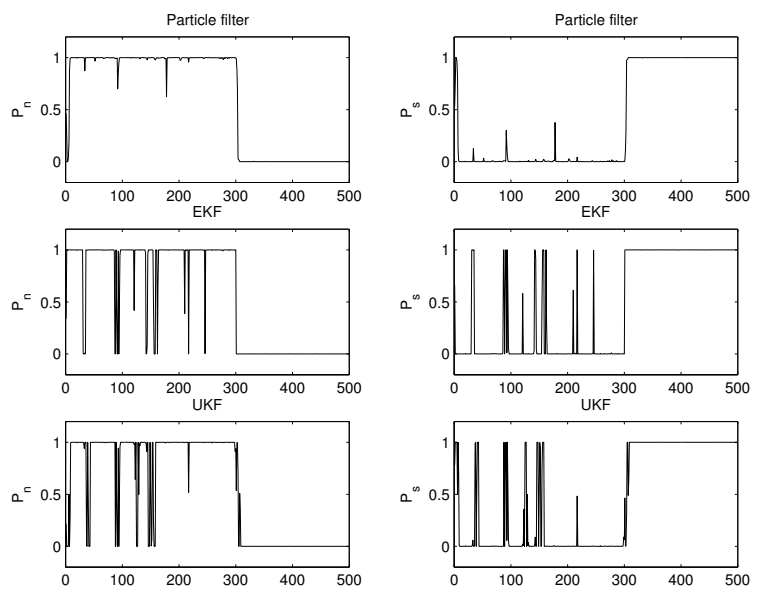

Fig. 5. Mode-conditional probability at each time step for EKF, UKF and particle filtering-based approaches
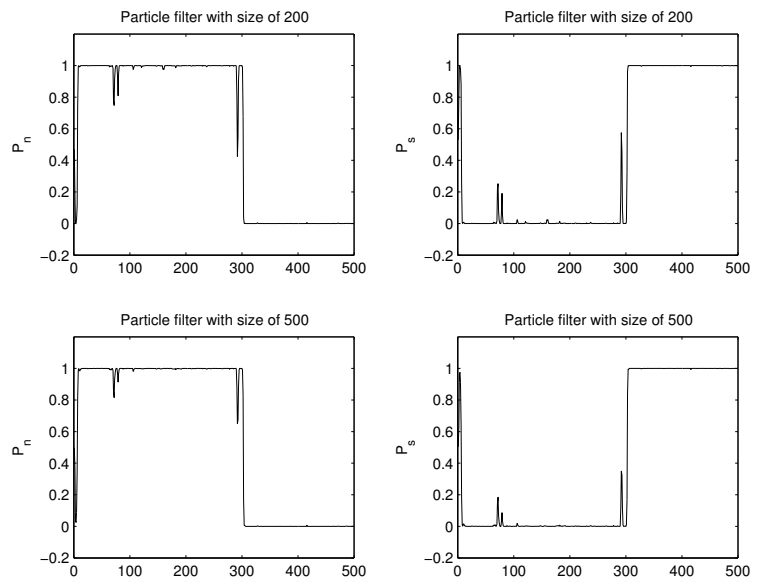

Fig. 6. Mode-conditional probability at each time step for particle filter with size of 200 and 500 determined, but also provide a complete representation of the posterior distribution of the state so that the various estimates of the state can be easily derived. The results from simulations show clearly that the fault diagnosis performance of the proposed particle filtering-based approach is superior to EKF and UKF-based fault diagnostic schemes. For the interacting multiple particle filtering algorithm, large sample size of particles is not necessary when the computation power is limited. Estimating faults in terms of a probability distribution over all possible system modes captures the uncertainty in fault identification that results form noisy and insufficient data.

\section{REFERENCES}

[1] Mehra, R. and Peschon, J., "An Innovation Approach to Fault Detection and Diagnosis in Dynamic Systems", Automatica, 7:637640, Pergamon Press, 1971.

[2] Willsky, A., "A survey of Design Methods for Failure Detection", Automatica., Nov. 1976, pp. 601-611.

[3] Kerr, T., "Real-time failure detection: A static nonlinear optimization problem that yields a two ellipsoid overlap test", Journal of Optimization Theory and Applications, Vol. 22, August 1977.

[4] Kerr, T., "Statistical Analysis of a Two-Ellipsoid Overlap Test for RealTime Failure Detection", IEEE Transaction on Automatic Control, Vol. AC-25, No. 4 August 1980.

[5] Skormin, V. A., Apone, J. and Dunphy, J. J., "On-line diagnostics of a self-contained flight actuator", IEEE Transactions on Aerospace and Electronic Systems, Vol. 30, No. 1, 1994, pp.186-196.

[6] Zhang, Y. M. and Li, X. R., "Detection and diagnosis of sensor and actuator failures using IMM estimator", IEEE Transactions on Aerospace and Electronic Systems, Vol 34, No. 4, 1998, pp.1293-1313.

[7] Jaw L. C., "Neural network for model-based prognostics", Proceedings of the IEEE Aerospace Conference, Vol. 3, 1999, pp.21-28.

[8] Hanlon, P. D. and Maybeck, P. S., "Multiple-model adaptive estimation using a residual correlation kalman filter bank", IEEE Transactions on Aerospace and Electronic Systems, Vol 36, No. 2, 2000, pp.393-406.

[9] Laparo, K. A., Buchner, M. C., and Vasudeva, K. S., "Leak detection in an experimental heat exchanger process: A multiple model approach", IEEE Transactions on Automatic Control, Vol 36, No. 2, 1991, pp.167-177.

[10] Menke, T.E., and Maybeck, P. S., "Sensor/actuator failure detection in the Vista F-16 by multiple model adaptive estimation", IEEE Transactions on Aerospace and Electronic Systems, Vol 31, No. 4, 1995, pp.393-406.

[11] Henk A. P. Blom and Yaakov Bar-shalom, "The interacting multiple model algorithm for systems with Markovian switching coefficients", IEEE Transactions on Automatic Control, Vol. 33, No. 8, 1988, pp.780-783.

[12] Zhang, Y. M. and Jiang, J., "Integrated active fault-tolerant control using IMM approach", IEEE Transactions on Aerospace and Electtronic Systems, Vol. 37, No. 4, 2001, pp.1221-1235.

[13] Mehra, M., Seereeram, S., Bayard, D. and Hadaegh, F., "Adaptive Kalman filtering, failure detection and identification for spacecraft attitude estimation", in Proceedings of the $4^{\text {th }}$ IEEE Conference on Control Applications, 1995, pp.176-181.

[14] Mehra, M. Rago, C. and Seereeram, S. "Autonomous failure detection, identification and fault-tolerant estimation with aerospace applications", in Proceedings of IEEE Aerospace Conference, Vol. 2, 1998, pp.133-138.

[15] Julier, S. J. and Uhlmann, J. K., "A new extension of the Kalman filter to nonlinear systems", in SPIE Aerospace Symposium, 1997.

[16] Jazwinski, A. H., "Stochastic processes and filtering theory", Academic Press, New York, 1970.

[17] Arulampalam, M. S., Maskell, S., Gordon, N., and Clapp T., "A tutorial on particle filters for online nonlinear/non-Gaussian Bayesian tracking”, IEEE Transactions on Signal Processing, Vol. 50, No. 2, 2002, pp. 174-188.

[18] Gordon, N. J. Salmond, D. J., and Smith, A. F. M., "Novel approach to nonlinear/non-Gaussian Bayesian state estimation", IEEE Proceedings-F, Vol. 140, No. 2, 1993, pp. 107-113. 